\title{
Escala para mensuração de modelo de negócios
}

\author{
Measurement scale for business models
}

\author{
Elnivan Moreira de Souza ${ }^{1}$ \\ Paulo César de Sousa Batista² \\ Márcio de Oliveira Mota ${ }^{3}$
}

\section{Resumo}

Este trabalho teve como objetivo construir e validar uma escala para mensurar as variáveis que definem o conceito de modelos de negócios. Utilizou-se como base teórica principal para a construção das escalas as contribuições de Osterwalder (2004) e Osterwalder e Pigneur (2010) sobre modelo de negócios. O caminho metodológico percorrido para os procedimentos de validação das escalas foi o sugerido por Costa (2011). A escala testada reunia 34 variáveis, agrupadas em quatro pilares: produto/serviço, interface com o cliente, gestão da infraestrutura e gestão financeira. O resultado do trabalho, uma contribuição inédita, foi a escala com 29 variáveis distribuídas nesses quatro pilares.

Palavras-chave: Modelo de Negócios. Validação de Escala. Mensuração.

\section{Abstract}

This paper proposes do build and validate a scale in order to measure variables that define the concepts of business models. The theoretical backgrounds that supported our proposed scale were based on Osterwalder (2004) andOsterwalderandPigneur

1 Mestre em Administração pela Universidade Estadual do Ceará. Professor da Universidade Estadual do Ceará (UECE) e do Centro Universitário Christus (UNICHRISTUS). Av. Silas Munguba, 1700, Campus do Itaperi, Fortaleza, Ceará, CEP: 60.714-903. E-mail: elnivan@ hotmail.com Fone: (85) 3101-9940 / 8614-9246.

$2 \mathrm{PhD}$ em Economia pela Universidade de Illinóis. Professor adjunto da Universidade Estadual do Ceará (UECE). Av. Silas Munguba, 1700, Campus do Itaperi, Fortaleza, Ceará, CEP: 60.714903. E-mail: batista.pcs@gmail.com Fone: (85) 3101-9940 / 8899-8675.

3 Doutor em Administração de Empresas pela Universidade de Fortaleza (UNIFOR). Professor da Universidade Estadual do Ceará (UECE). Av. Silas Munguba, 1700, Campus do Itaperi, Fortaleza, Ceará, CEP: 60.714-903. E-mail: marcio@marciomota.com Fone: (85) 3101-9940/ 8899-6654. 
(2010) and our methodology design was based on Costa (2011). The proposed scale is grouped into four bases: Product/Service, Embeddedness with the Customers, Resources Management and Financial Management. The outcomes contributed to the academia with a business model with 29 variables grouped into these four bases.

Keywords: Business model. Scale validation. Measurement.

\section{Introdução}

Aexpressão "modelo de negócios" tem sido utilizada com frequência por consultores, executivos e acadêmicos, principalmente depois que surgiram os negócios baseados na internet (OSTERWALDER; PIGNEUR, 2013). Mesmo assim, o constructo "modelo de negócios" não possui uma definição clara e inequívoca na literatura, o que tem dificultado os estudos sobre o tema. Zhu, Kramer e Xu (2006) realçaram que o impacto econômico dos negócios eletrônicos (e-business) e o seu potencial para transformar modelos de negócios, estruturas organizacionais, processos, relações com os consumidores e fornecedores são de conhecimento universal.

A internet evoluiu da condição de ferramenta de disseminação de informações e de trocas econômicas on-line para se tornar o próprio modelo de negócios das empresas. Estas se transformaram criando ou adaptando seu modelo de negócios de tal modo que há uma interação global das atividades organizacionais. Nesse contexto, várias pesquisas foram realizadas no intuito de entender a dinâmica das atividades que compõem o modelo de negócios adotado pelas empresas (CHASTON; MAGLES, 2002;OSTERWALDER, 2004; OSTERWALDER; PIGNEUR, 2013; OSTERWALDER; PIGNEUR; TUCCI, 2005;WU; MAHJAN; BALASUBRAMANIAN, 2003).

De todas as contribuições analisadas, encontram-se entre as de maior pertinência, segundo levantamento bibliométrico de Zott, Amitt e Massa (2011), as de Osterwalder, Pigneur e Tucci (2005), que vêm sendo largamente difundidas no meio profissional. Esses autores desenvolveram uma ferramenta (Canvas) de análise sustentada em nove dimensões, alocadas em quatro pilares: produto, interface com o cliente, 
gestão da infraestrutura e gestão financeira. Com essa ferramenta, estudaram empresas como Dell, Amazon.com e eBay.

No Brasil, Araújo e Zilber (2013) utilizaram os conceitos dessa ferramenta numa pesquisa realizada com pequenas empresas de e-business da cidade de São Paulo. Santos, Seloti e Silva (2013) aplicaram-na para estudar o modelo de negócios de panificadoras tradicionais e gourmets. A abordagem de Araújo e Zilber (2013), por exemplo, teve na sua etapa metodológica o desenvolvimento de variáveis, porém, os constructos e conceitos utilizados para mediação são diferentes dos empregados nesta pesquisa. Essas contribuições são relevantes, mas não tiveram em seus desdobramentos o desenvolvimento de uma escala para analisar as relações dos constructos desse modelo pelo uso de técnicas multivariadas. Zott, Amit e Massa (2011), em estudo bibliométrico sobre o uso do termo "modelo de negócios", indicaram a carência de estudos quantitativos sobre o conceito. Visando preencher essa lacuna, este estudo objetiva construir e validar uma escala para mensurar as variáveis que definem o conceito de modelo de negócios.

No cumprimento desses objetivos, a survey é adotada como estratégia da pesquisa, que utiliza questionários impressos e eletrônicos, com escalas Likert de cinco pontos. Para a análise dos dados, são utilizadas a análise descritiva e a análise multivariada de dados, esta última compreendida por análise fatorial exploratória e procedimentos próprios para validação de escala (COSTA, 2011). O universo da pesquisa compreendeu gestores, empreendedores e demais profissionais engajados em atividades que utilizam as tecnologias hospedadas na internet para fazer negócios. A amostra é não probabilística e se constituiu de 252 casos.

Além desta introdução, este trabalho é composto de mais três seções e das considerações finais. A segunda seção consiste na fundamentação teórica, a terceira contempla os aspectos metodológicos, a quarta aborda a análise dos resultados e, por fim, há as considerações finais do estudo. 


\section{Fundamentação teórica}

Segundo Osterwalder e Pigneur (2013), os conceitos associados ao uso dos modelos de negócios vêm ganhando espaço na agenda de consultores, executivos e acadêmicos, principalmente depois que despontaram os negócios baseados na internet. Uma das dificuldades no estudo dos modelos de negócios é que o constructo não possui uma definição clara e inequívoca na literatura.

Acadêmicos como Amit e Zott (2001) e Rappa (2004) têm se empenhado em definir esse constructo para adequar seu uso à prática empresarial, a partir de teorias, ferramentas e conceitos associados à estratégia e às vantagens competitivas, como a visão baseada em recursos, a análise da cadeia de valor, a teoria da rede estratégica e a economia dos custos de transação. Amitt e Zott (2001) mencionam ainda que o uso de tecnologias acaba por influenciar a estratégia e a arquitetura organizacional das empresas. Outros autores, como Chesbrough (2010), Ethiraj, Guler e Singh (2000), Mansfield e Fourie (2004) e Seddon et al. (2004) admitem que a estratégia e o modelo de negócios se sobrepõem, pois o segundo constructo é uma metáfora da estratégia.

Quase todos os autores citados têm definido modelo de negócios como um novo constructo nos campos da administração e da tecnologia da informação (CHESBROUGH, 2010; MAGRETTA, 2002; SEDDON, 2004). Para Osterwalder, Pigneur e Tucci (2005), o modelo de negócios é um plano em construção que permite projetar e realizar a estrutura de negócios e sistemas, constituindo a forma operacional e física que a empresa vai tomar. É formado pela triangulação entre estratégia, organização e os sistemas de informação que constantemente recebem pressão dos ambientes sociais e legais, além das forças competitivas, da demanda dos consumidores e das mudanças tecnológicas (Figura 1). 


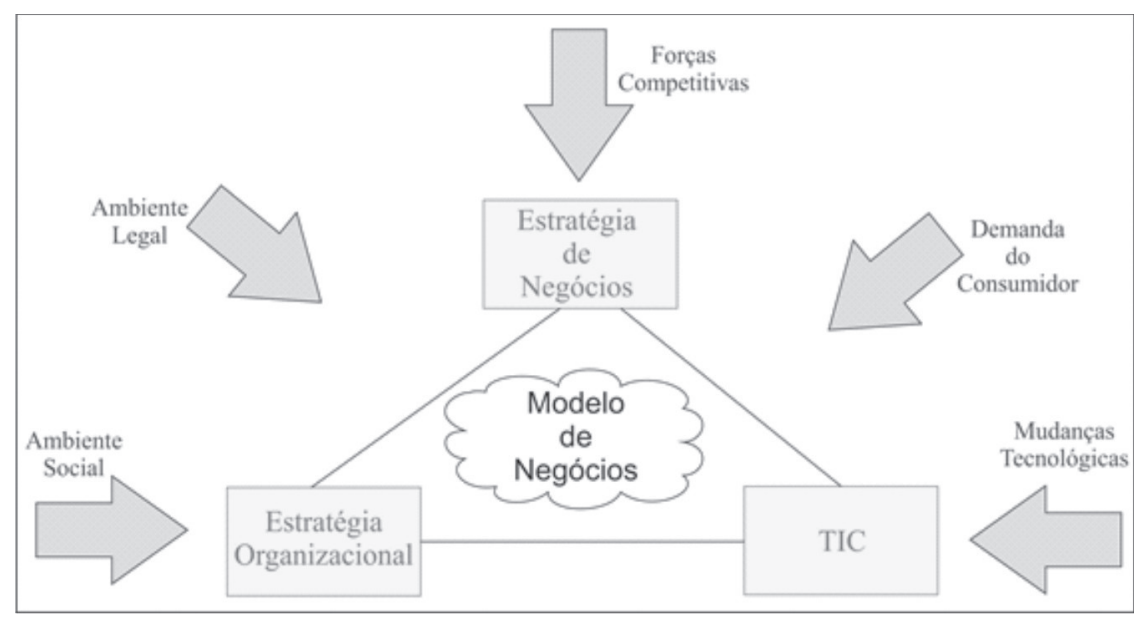

Fonte: Osterwalder, Pigneur e Tucci (2005)

Figura 1 - Ambiente, Modelo de Negócios, Estratégia e Sistemas de Informação.

A construção do modelo de negócios possibilitará às empresas implantar as atividades alinhadas ao uso das tecnologias de informação e comunicação (TIC), sendo estas definidas como a fusão complexa de processos comerciais, aplicações empresariais e estrutura organizacional necessária para criar um negócio de alto desempenho. Uma estratégia integrada deve direcionar os investimentos requeridos para desenvolver a infraestrutura necessária não só em termos de sistemas de informação, mas principalmente de recursos humanos e processos adequados para fundamentar as operações. Nessas circunstâncias, o conceito de modelo de negócios como orientador da arquitetura da empresa para entrega de valor é relevante.

Joia e Ferreira (2005), ao estudarem as diferenças práticas e conceituais entre modelos de negócios e estratégia, chegaram ao entendimento de que o primeiro é um instrumento dinâmico e sistêmico para várias abordagens do segundo. Isso possibilita entrelaçá-los em uma definição, aumentando o potencial dessas abordagens para explicar e orientar o processo estratégico real das organizações. Será esse o conceito de modelo de negócios que embasará o presente trabalho. 
Osterwalder (2004) e Osterwalder, Pigneur e Tucci (2005) propõem um framework para formulação dos modelos de negócios de qualquer natureza, embora enfatizem que seu modelo é o mais adequado para empresas intensivas no uso de tecnologias porque busca alinhar estratégias empresariais, organizacionais e tecnológicas. O modelo de negócios proposto é composto por quatro pilares, que se dividem em nove dimensões constituintes do escopo de atividades das empresas: (I) proposta de valor; (II) segmentação de mercado; (III) canais de distribuição; (IV) relacionamento com o cliente; (V) capacidades; (VI) configuração de valor; (VII) parcerias; (VIII) modelo de receitas; e (IX) estrutura de custos (FIG 2).

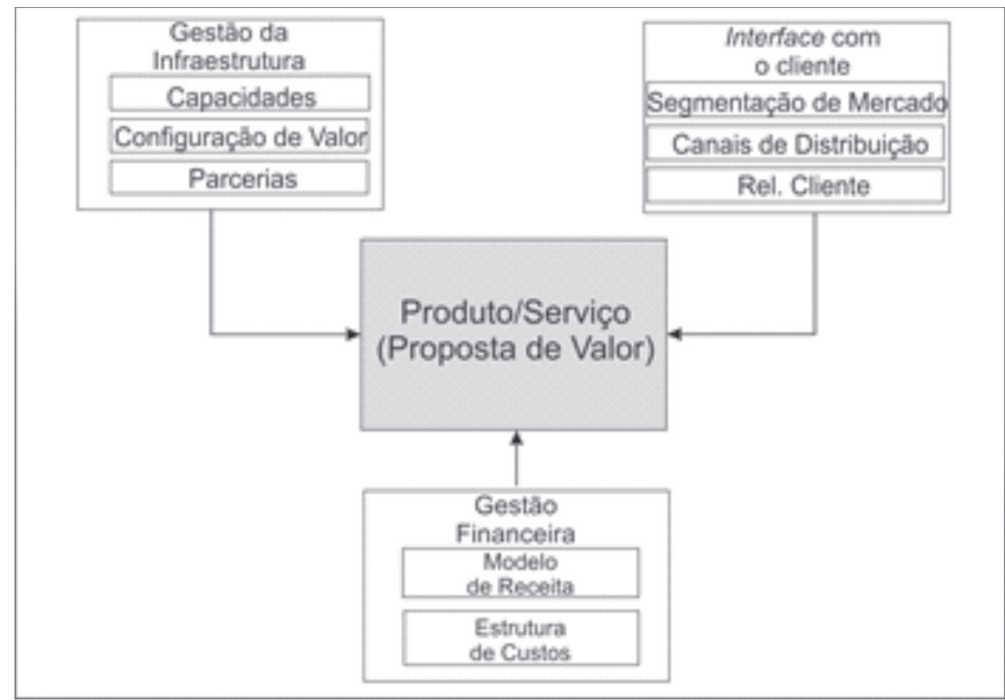

Fonte: Baseado em Osterwalder, Pigneur e Tucci (2005)

Figura 2 - Modelo de Negócios Intensivos em Tecnologia

Em 2010, com a coautoria de Yves Pigneur e a ajuda de 470 profissionais e acadêmicos de 45 países, Osterwalder publicou o livro Business Model Generation, traduzido no Brasil como Modelo de Inovação em Negócios, que apresenta a ferramenta Canvas, com o propósito de auxiliar os empreendedores e gestores na formulação 
de seus modelos de negócios. Nos últimos anos, essa ferramenta foi bastante disseminada e utilizada por profissionais e consultores, embora sem a preocupação de validar empiricamente suas relações implícitas.

No Brasil, exemplos da aplicação dessa ferramenta em pesquisas são encontrados em Araújo e Zilber (2013) e Santos, Seloti e Silva (2013), autores que igualmente não verificaram empiricamente as relações implícitas no modelo. As dimensões integrantes do framework proposto por esses autores são apresentadas a seguir e servem de base para a construção da escala do modelo de negócios.

O produto/serviço é o pilar central no Modelo Ontológico de Negócios proposto por Osterwalder, Pigneur e Tucci (2005), pois expressa a proposta de valor apresentada pela empresa ao mercado e condiciona todas as atividades a serem realizadas. A proposta de valor apresentada no modelo de negócios pode ser entendida como a declaração dos benefícios a serem entregues pela empresa aos seus clientes e demais stakeholders (BAGCHI; TULSKIE, 2000). Compreendem essa arquitetura de valor a utilidade para o cliente, a inovação, a excelência, o preço, a liquidez e o ciclo de vida do produto. O segundo bloco do Modelo, constituído pela interface com o cliente, integra três dimensões: (I) segmentação de mercado, (II) canais de distribuição e (III) relacionamento com o cliente. Essa área referese à maneira como a empresa se apresenta ao mercado, como ela realmente atinge e interage com seus clientes (OSTERWALDER, 2004; OSTERWALDER; PIGNEUR, 2010).

De acordo com Osterwalder e Pigneur (2010), a segmentação eficaz permite que uma empresa aloque melhor os recursos de investimento para os clientes-alvo que serão mais atraídos pela sua proposta de valor, além de auxiliar no processo de decisão de escolhas dos canais de distribuição. A distinção genérica dos clientes-alvo existe entre as empresas e/ou clientes individuais, comumente referidos como business-to-business (B2B) e business-to-consumer (B2C).

O canal de distribuição corresponde ao meio pelo qual a organização consegue entrar em contato com o cliente. São as atividades 
dessa dimensão que irão interligar a proposta de valor à segmentação de mercado. Sua finalidade é fazer chegar aos clientes as quantidades certas de produtos certos ou serviços disponíveis na renda certa, no momento certo, para as pessoas certas (PITT; BERTHON; BERTHON, 1999), sujeito às restrições de custo, investimento e flexibilidade (ANDERSON; DAY; RANGAN, 1997).

As atividades do relacionamento com o cliente definem o tipo de ligação que a empresa possui com o cliente, bem como a força do relacionamento que a organização quer com eles (OSTERWALDER; PIGNEUR; TUCCI, 2005). As empresas devem analisar os dados dos clientes, a fim de avaliar o tipo de cliente que querem encantar e adquirir, quais são rentáveis e quais valem a pena os esforços de retenção de gastos e são susceptíveis de aumentar as vendas (BLATTBERG; GETZ; THOMAS, 2001).

Agestão da infraestrutura é o terceiro bloco do Modelo e descreve as habilidades necessárias para promover a proposta de valor, envolvendo parcerias, capacidades e configuração de valor. Como as ligações entre as empresas estão cada vez mais eletrônicas, os membros de uma rede são flexíveis na coordenação de horários, compartilhamento de ativos, competências e recursos, bem como cooperam no desenvolvimento, realização e fechamento de negócios (ANDREWS; HAHN, 1998).

Wallin (2006) descreve as capacidades como padrões repetitivos de ação na utilização dos ativos para criar, produzir e ofertar produtos e serviços para o mercado. Assim, uma empresa precisa dispor de um conjunto de recursos, a fim de prestar a sua proposta de valor (WERNEFELT, 1984). Nesse sentido, Grant (1991) faz uma distinção entre ativos tangíveis e intangíveis, e habilidades baseadas nas pessoas. Recursos tangíveis incluem plantas, equipamentos e reservas de dinheiro. Recursos intangíveis incluem patentes, copyrights, reputação, marcas e segredos comerciais. Os recursos humanos são as pessoas que uma empresa precisa para criar valor com os recursos tangíveis e intangíveis. 
A configuração do valor integra, a fim de criar valor para o cliente, todas as atividades necessárias e as ligações entre elas, que são agrupadas em três tipos básicos: a cadeia de valor (PORTER, 2001), o valor de compra e o valor da rede (STABELL; FJELDSTAD, 1998). A cadeia de valor contém as diferentes atividades que uma empresa realiza para oferecer produtos de baixo custo ou diferenciados. $O$ valor de compra representa uma extensão do framework da cadeia de valor de Porter (2001). No valor de rede, o valor é criado por meio da ligação entre clientes (STABELL; FJELDSTAD 1998).

A parceria, terceira dimensão da gestão da infraestrutura, é uma iniciativa voluntária acordada entre duas ou mais organizações com o objetivo de criar valor para os clientes. No contexto do e-business e seus correlatos, há várias denominações para essas novas formas de redes estratégicas no processo de criação de valor, dentre elas, o b-webs (TAPSCOTT; TICCOLL; LOWI, 2000), as organizações de fluidos e flexíveis (SELZ, 1999), e as redes de valor (NALEBUFF; BRANDENBURGER, 1997). As empresas entram em parcerias por três categorias de motivações: otimização e economia de escala; redução do risco e incertezas; e aquisição de recursos (OSTERWALDER, 2004).

A gestão financeira é o quarto bloco do framework proposto por Osterwalder (2004), compreendendo o modelo de receitas e a estrutura de custos. Juntos, eles determinam a situação financeira da empresa e sua habilidade de sobrevivência no mercado competitivo.

O modelo de receitas mede a capacidade de uma empresa para traduzir o valor que ele oferece aos seus clientes em dinheiro e fluxos de receitas recebidas, composto por diferentes modalidades: comissões, patentes, juros de empréstimos, aluguéis etc. $\mathrm{O}$ valor e a sustentabilidade do negócio são determinados pelo modelo de receitas (OSTERWALDER, 2004; OSTERWALDER; PIGNEUR, 2010).

A estrutura de custos mede todos os custos gerados pela empresa, a fim de criar, comercializar e entregar valor aos seus clientes. Ele define preço para todos os recursos, bens, atividades e relacionamentos da rede de parceiros e intercâmbios (OSTERWLADER 2004). 


\section{Aspectos metodológicos}

Esta seção apresenta os procedimentos e as decisões metodológicas seguidas no desenvolvimento da investigação empírica. Para a consecução do objetivo principal do trabalho, recorreu-se a duas fases distintas de investigação.

Na primeira, denominada fase exploratória, procedeu-se a uma considerável revisão de literatura, com o intuito de construir bases teóricas consistentes para os tópicos abordados no estudo, a fim de permitir o mapeamento dos constructos e sua forma de operacionalização. Com a adoção desses procedimentos, foram identificadas nove dimensões relacionadas à arquitetura e à dinâmica dos modelos de negócios, a saber:

- Proposta de Valor (PS_PV);

- Segmentação de Mercado (IC_SM);

- Canais de Distribuição (IC_CD);

- Relacionamento com o Cliente (IC_RC);

- Capacidades (GI_CP);

- Configuração de Valor (GI_CV);

- Parcerias (GI_PA);

- Modelo de Receitas (GF_MR);

- Estrutura de Custos (GF_EC).

As delimitações desses constructos serviram de base para a construção do instrumento de pesquisa, um questionário estruturado, composto por 34 afirmativas mensuradas por meio de uma escala de Likert de cinco pontos, além de mais oito questões relacionadas a informações gerais, perfil sociodemográfico dos respondentes e suas respectivas empresas.

Após os procedimentos preliminares de ajuste do instrumento, de avaliação por pesquisadores especialistas e de pré-teste, o questionário foi aplicado com 252 gestores, empreendedores e demais profissionais engajados em atividades que dependem do uso intensivo de tecnologia. A amostra, não probabilística, foi definida por acessibilidade, e a coleta 
de dados foi realizada nos meses de novembro e dezembro de 2013, por meio de abordagem direta ou pelo uso de questionários eletrônicos nas redes sociais.

Na segunda fase da pesquisa, os dados foram analisados com o auxílio do software Statistical Package for the Social Sciences (SPSS) para Análises Descritivas, testes não paramétricos, correlações de Pearson, testes de confiabilidade e análise fatorial exploratória (AFE). O uso da AFE fez-se necessário por não ter sido encontrado teste estatístico que trouxesse uma predefinição dos constructos, sugeridos com base em estudos qualitativos, meta-análise e revisão de literatura.

Os missings foram tratados pelo procedimento de substituição dos valores ausentes através do método da tendência linear do ponto, e os outliers foram considerados observações extraordinárias inexplicáveis por se tratarem de percepções subjetivas dos respondentes em relação às empresas nas quais trabalham (HAIR et al., 2009). Os índices de não resposta variaram de $1 \%$ a $4 \%$, estando, portanto, dentro da faixa recomendada pela literatura especializada, que é de até 10\% (COSTA, 2011).

Todo o procedimento para elaboração, adaptação e validação das escalas foi realizado conforme orientações de Costa (2011): a) especificação do domínio do constructo; b) atividades de geração de itens e validação de face e conteúdo; c) decisões sobre as respostas; d) construção do instrumento de pesquisa; e) primeira atividade de amostragem; f) primeiros procedimentos de limpeza da escala; g) atividades de campo adicionais; h) procedimentos de limpeza da escala adicionais; i) análise de validade e de confiabilidade da escala final; e j) desenvolvimento de normas e recomendações de uso e interpretação.

\section{Análise dos resultados}

A análise foi realizada com 252 entradas de dados. A Tabela 1 apresenta o perfil dos respondentes, com indicadores de gênero, idade e cargo que exercem. 
Tabela 1: Dados Demográficos dos Respondentes

\begin{tabular}{lcc}
\hline Perfil & Frequência & Porcentagem (\%) \\
\hline Gênero & 171 & 67,9 \\
Masculino & 80 & 31,7 \\
Feminino & 1 & 0,4 \\
Missings & & \\
Idade & 22 & 8,7 \\
$<20$ & 155 & 61,5 \\
$21-30$ & 40 & 15,9 \\
$31-40$ & 21 & 8,3 \\
$41-50$ & 5 & 2,0 \\
$51-60$ & 4 & 1,6 \\
61 ou mais & 5 & 2,0 \\
Missings & & \\
Cargo & 31 & 12,3 \\
Estagiário & 61 & 24,2 \\
Outros & 48 & 19,0 \\
Supervisão & 55 & 21,8 \\
Gerência & 55 & 21,8 \\
Proprietário & 2 & 0,80 \\
Missings & &
\end{tabular}

Fonte: Dados da pesquisa

São necessárias algumas observações em relação aos dados demográficos dos respondentes, como aproximação do conceito de modelo de negócios e qualificação dos dados coletados. Por exemplo, quanto à idade, $61,5 \%$ dos respondentes estão na faixa etária entre $21 \mathrm{e}$ 30 anos. Caso seja considerado o intervalo que compreende de menos 20 até 40 anos, há uma concentração de $86,1 \%$ dos respondentes, e esse perfil engloba profissionais que se enquadram nas gerações $Y$ (que cresceu e acompanhou o desenvolvimento das tecnologias de internet) e $\mathrm{Z}$ (que atualmente vive a era das redes sociais e do mobile) (SERRANO, 2010). 
Quanto ao cargo dos respondentes, mais de $60 \%$ da amostra é composta por supervisores, gerentes e proprietários de empresas, distribuídos nos mais variados portes.

A Tabela 2 apresenta o perfil das empresas dos respondentes, compreendendo informações como setor de atividade, tempo da empresa no mercado e faturamento médio anual.

Tabela 2: Perfil das Empresas dos Respondentes

\begin{tabular}{lcc}
\hline Perfil & Frequência & Porcentagem (\%) \\
\hline Setor de Atividade & & \\
Comércio & 68 & 27,0 \\
Indústria & 43 & 17,1 \\
Serviço Online & 27 & 10,7 \\
Serviço Financeiro & 20 & 7,9 \\
Serviço Tradicional & 52 & 20,6 \\
Publicidade & 5 & 2,0 \\
Outro & 34 & 13,5 \\
Missings & 3 & 1,2 \\
Tempo da Empresa no Mercado & & \\
0 a 5 anos & 80 & 31,7 \\
6 a 10 anos & 40 & 15,9 \\
10 a 20 anos & 41 & 16,3 \\
$>$ 20 anos & 84 & 33,3 \\
Missings & 7 & 2,8 \\
Faturamento Anual (R\$) & & \\
Até 60.000,00 & 40 & 15,9 \\
$60.000,01-360.000,00$ & 33 & 13,1 \\
360.000,01-3.600.000,00 & 54 & 21,4 \\
3.600.000,01a12.000.000,00 & 37 & 14,7 \\
> 12.000.000,00 & 74 & 29,4 \\
Missings & 14 & \\
\hline
\end{tabular}

Fonte: dados da pesquisa 
Com relação ao perfil das empresas, mais especificamente o setor de atuação, optou-se por desmembrar a categoria "Serviços" em três, em decorrência da maior variedade de atividades. As categorias "Serviços On-line" e "Serviço Financeiro" são de fato mais afetadas pela incorporação de tecnologias de internet/e-business em seus processos usuais de gestão, portanto, detentoras da concepção de modelo de negócios.

\subsection{Procedimentos de validação e construção da escala}

O procedimento de limpeza das escalas envolveu quatro atividades: análise exploratória preliminar, análise de correlação, análise fatorial exploratória e análise de confiabilidade. A análise exploratória preliminar consistiu na avaliação visual, tratamento de missings e ouliers, procedimentos já demonstrados em seções anteriores. Para análise das escalas, observam-se os seguintes critérios (COSTA, 2011, HAIR et al., 2009):

- A análise de correlação de Pearson, 0,2>0,9, com significância ao nível de $5 \%$;

- A análise fatorial exploratória com comunalidades maiores que 0,4 , teste de esfericidade de Bartlett significativo ao nível de $5 \%$, Kaiser-Meyer-Olkin (KMO) acima de 0,7 e engenvalues acima de 1 ;

- A análise de confiabilidade deve apresentar um Alfa de Cronbach superior a 0,60.

A maioria das variáveis agrupou-se em constructos teóricos conforme o Quadro 1. 
Quadro 1: Construtos e Variáveis Propostas para Modelo de Negócios

\begin{tabular}{|c|c|}
\hline & PRODUTO/SERVIÇO (PS) \\
\hline & PROPOSTA DE VALOR (PV) \\
\hline PS_PV1 & $\begin{array}{l}\text { O produto/serviço que a empresa oferece atende perfeitamente a } \\
\text { necessidade dos clientes }\end{array}$ \\
\hline PS_PV2 & $\begin{array}{l}\text { Os produtos/serviços oferecidos pela empresa são conhecidos por } \\
\text { sua qualidade }\end{array}$ \\
\hline PS_PV3 & $\begin{array}{l}\text { O preço do produto/serviço da empresa exerce impacto relevante na } \\
\text { proposta de valor }\end{array}$ \\
\hline PS_PV4* & A empresa oferece um produto/serviço considerado inovador \\
\hline \multirow[t]{4}{*}{ PS_PV5* } & $\begin{array}{l}\text { O ciclo de vida do produto/serviço oferecido pela empresa é } \\
\text { relativamente curto (menos de } 1 \text { ano) }\end{array}$ \\
\hline & $\begin{array}{l}\text { Base Teórica: Bagchi, Tuldkie (2000); Osterwalder (2004); Osterwalder, } \\
\text { Pirneur, Tucci (2005); }\end{array}$ \\
\hline & INTERFACE COM O CLIENTE (IC) \\
\hline & SEGMENTAÇÃO DE MERCADO (SM) \\
\hline IC_SM1 & $\begin{array}{l}\text { Na empresa há uma definição clara do segmento de mercado que ela } \\
\text { atende }\end{array}$ \\
\hline IC_SM2 & $\begin{array}{l}\text { O segmento de mercado que a empresa atende está diretamente } \\
\text { ligado ao produto/serviço que ela oferece }\end{array}$ \\
\hline \multirow[t]{3}{*}{ IC_SM3 } & $\begin{array}{l}\text { A empresa já é reconhecida no mercado por conta do produto/serviço } \\
\text { que oferece }\end{array}$ \\
\hline & Base Teórica:Ostewalder, Pigneur (2010). \\
\hline & CANAIS DE DISTRIBUIÇÃO (CD) \\
\hline IC_CD1 & A empresa utiliza a internet para entregar produtos/serviços \\
\hline IC_CD2 & $\begin{array}{l}\text { A empresa utiliza mais de um mecanismo para entregar produtos/ } \\
\text { serviços }\end{array}$ \\
\hline IC_CD3 & $\begin{array}{l}\text { As operações da empresa são integradas com a logística (entrega de } \\
\text { produtos) }\end{array}$ \\
\hline \multirow[t]{2}{*}{ IC_CD4 } & $\begin{array}{l}\text { O tipo de cliente que a empresa atende é considerado para a escolha } \\
\text { dos canais de distribuição }\end{array}$ \\
\hline & $\begin{array}{l}\text { Base Teórica: Anderson, Day e Rangan (1997) e Pitt, Berthon, Berthon } \\
\text { (1999). }\end{array}$ \\
\hline
\end{tabular}




\begin{tabular}{|c|c|}
\hline & RELACIONAMENTO COM O CLIENTE (RC) \\
\hline IC_RC1 & A empresa adota um relacionamento personalizado com os clientes \\
\hline IC_RC2 & Os clientes têm confiança na empresa/marca \\
\hline IC_RC3* & $\begin{array}{l}\text { A relação com os clientes é baseada em elevados custos de mudanças } \\
\text { (ex. substituição de software) }\end{array}$ \\
\hline \multirow[t]{4}{*}{ IC_RC4 } & $\begin{array}{l}\text { A empresa consegue reter e manter um bom relacionamento com os } \\
\text { clientes }\end{array}$ \\
\hline & $\begin{array}{l}\text { Base Teórica: Blattberg, Getz, Thomas (2001) e Osterwalder, Pigneur, } \\
\text { Tucci (2005) }\end{array}$ \\
\hline & GESTÃO DA INFRAESTRUTURA (GI) \\
\hline & CAPACIDADES (CP) \\
\hline GI_CP1 & $\begin{array}{l}\text { A empresa consegue usar bem os recursos que possui para oferecer } \\
\text { produtos/serviços diferenciados }\end{array}$ \\
\hline GI_CP2 & $\begin{array}{l}\text { A empresa sabe utilizar máquinas, prédios e equipamentos para gerar } \\
\text { produtos/serviços de qualidade }\end{array}$ \\
\hline \multirow[t]{3}{*}{ GI_CP3 } & $\begin{array}{l}\text { A empresa sabe utilizar o conhecimento e as habilidades da equipe } \\
\text { para gerar produtos/serviços inovadores }\end{array}$ \\
\hline & Base Teórica: Wallin (2006); Wernefelt (1984) e Grant (1991) \\
\hline & CONFIGURAÇÃO DE VALOR (CV) \\
\hline GI_CV1** & A empresa utiliza de forma integrada todas as atividades operacionais \\
\hline Gl_CV2** & A empresa busca agregar valor incorporando novos produtos/serviços \\
\hline \multirow[t]{3}{*}{ GI_CV3* } & Os produtos/serviços oferecidos pela empresa são difíceis de copiar \\
\hline & Base Teórica: Porter (2001) e Stabell e Fjeldstad (1998) \\
\hline & PARCERIAS (PA) \\
\hline GI_PA1 & $\begin{array}{l}\text { A empresa estabelece parcerias para adquirir produtos/serviços mais } \\
\text { baratos }\end{array}$ \\
\hline GI_PA2 & $\begin{array}{l}\text { A empresa forma parcerias para reduzir riscos e incertezas do } \\
\text { ambiente }\end{array}$ \\
\hline GI_PA3 & $\begin{array}{l}\text { A empresa forma parcerias para adquirir novos recursos como } \\
\text { máquinas e equipamentos }\end{array}$ \\
\hline \multirow[t]{2}{*}{ GI_PA4 } & $\begin{array}{l}\text { A empresa forma parcerias para adquirir novos recursos como } \\
\text { patentes, marcas e conhecimentos }\end{array}$ \\
\hline & $\begin{array}{l}\text { Base Teórica: Nalebuff, Brandenburger (1997); Osterwalder (2004); } \\
\text { Tapscott, Ticcoli, Lowi (2000). }\end{array}$ \\
\hline
\end{tabular}




\begin{tabular}{ll}
\hline \multicolumn{2}{c}{ GESTÃO FINANCEIRA (GF) } \\
\hline GF_MR1* & A empresa aufere receita por meio da venda de produtos/serviços \\
GF_MR2 & A empresa aufere receita pelo recebimento de juros de empréstimo \\
GF_MR3 & $\begin{array}{l}\text { A empresa aufere receita com a publicidade de produtos/serviços de } \\
\text { empresas parceiras }\end{array}$ \\
GF_MR4 & $\begin{array}{l}\text { A empresa aufere receita pelo recebimento de comissões } \\
\text { Base Teórica: Osterwalder (2004) e Osterwalder e Pigneur (2010). }\end{array}$ \\
GF_EC1 & $\begin{array}{l}\text { ESTRUTURA DE CUSTOS (EC) } \\
\text { GF_EC2 }\end{array}$ \\
A empresa sabe a participação de cada item da sua estrutura de custos \\
GF_EC3 & $\begin{array}{l}\text { Os custos da empresa estão compatíveis com os custos dos principais } \\
\text { concorrentes }\end{array}$ \\
GF_EC4 & $\begin{array}{l}\text { A estrutura de custos da empresa é compatível com o modelo de } \\
\text { negócio que ela adota }\end{array}$ \\
\cline { 2 - 3 } & Base Teórica: Osterwalder (2004).
\end{tabular}

*Excluída da escala final / ** Reagrupadas no constructo "Capacidades"

Fonte: Elaborado pelo autor com base em Osterwalder (2004); Osterwalder, Pigneur e Tucci (2005) e Osterwalder e Pigneur (2010).

Por não se enquadrarem nos critérios descritos acima, foram excluídas as seguintes variáveis: PS_PV4, PS_PV5, IC_RC3, GI_CV3 e GF_MR1, dentre as quais são ressaltadas as que deixaram de se agrupar ao constructo "Configuração de Valor", como esperado. 
Tabela 3: Indicadores Estatísticos de Validação da Escala para Modelo de Negócios

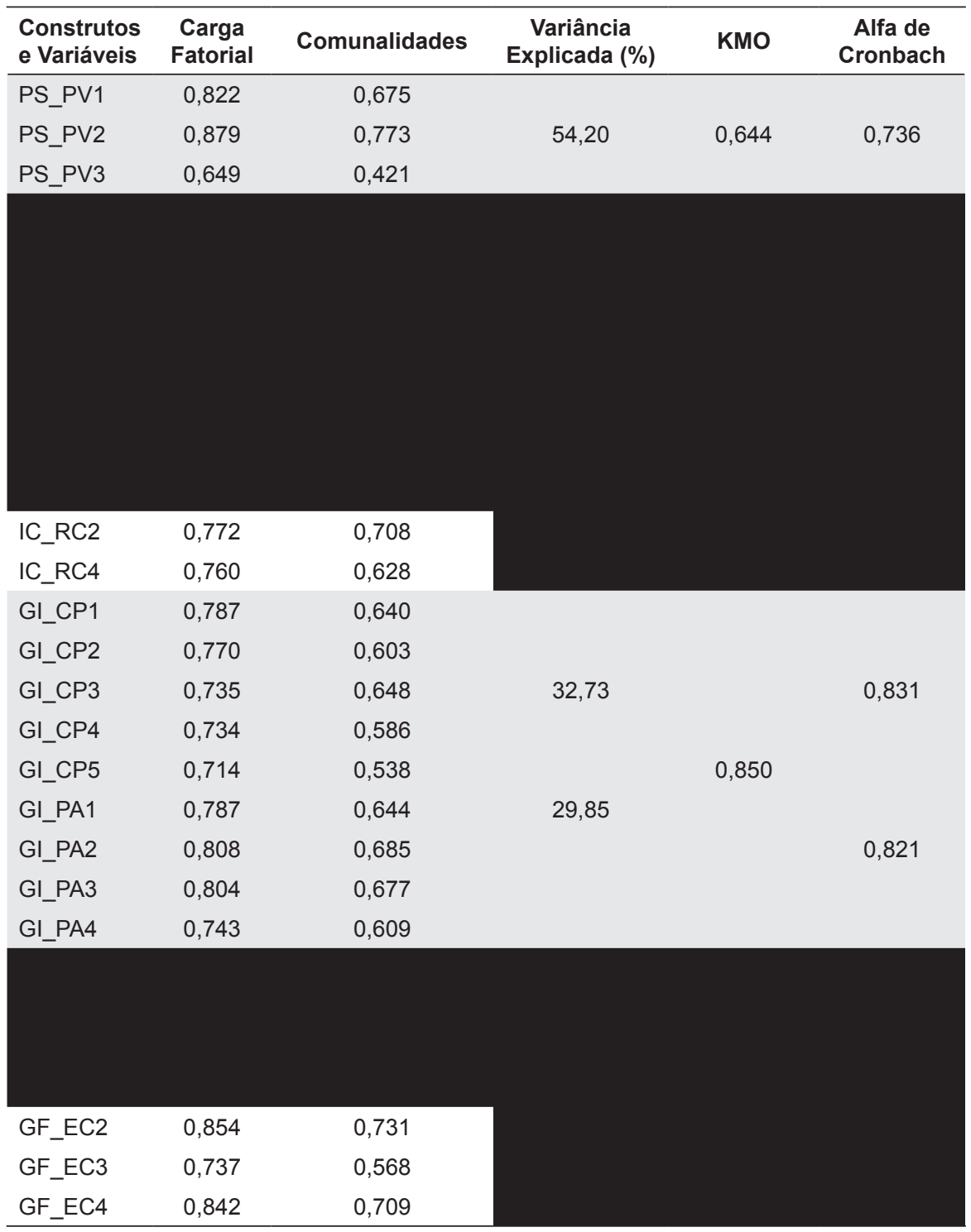

Fonte: Dados da pesquisa. 
A variável GI_CV3 foi retirada da escala por várias inconsistências, dentre as quais, baixa correlação e por não ter se agrupado a nenhum fator específico. As variáveis GI_CV1 e GI_CV2 retornaram bons indicadores, mas se agruparam ao constructo "Capacidades", quando se esperava seu enquadramento em "Configuração do Valor". Ao se analisar as afirmativas do constructo GI_CV, verifica-se que a variável GI_CV1 versa sobre a capacidade de a empresa utilizar de forma integrada todas as atividades operacionais. A GI_CV2 versa sobre a capacidade de a empresa agregar valor incorporando novos produtos/serviços. Portanto, verifica-se que essas afirmativas estão mais associadas às capacidades do que à configuração de valor. Sendo assim, o constructo GI_CV foi dissolvido. A variável GI_CV3 foi excluída, as variáveis GI_CV1 e GI_ CV2 foram incorporadas ao constructo GI_CP e passam a se chamar, respectivamente, GI_CP4 e GI_CP5.

Para verificação da validade discriminante, os fatores dos constructos foram transformados em variáveis e foram extraídas suas correlações. Todos os constructos apresentaram validade discriminante, conforme Costa (2011).

Na Tabela 3, apresentam-se os dados consolidados da escala validada para mensuração do modelo de negócios, com seus respectivos constructos, cargas fatoriais, comunalidades, variância explicada, KMO e Alfa de Cronbach.

Todos os indicadores apresentaram números que caracterizam sua validação nos termos dos critérios estabelecidos pelos manuais. Embora não apresentadas neste artigo, as correlações de Pearson foram calculadas e seus resultados também ficaram conforme as orientações de Hairet al. (2009).

\subsection{Implicações teóricas e empíricas da escala desenvolvida}

O desenvolvimento da escala para mensuração do modelo de negócios preenche uma lacuna encontrada na literatura pelos autores deste artigo, corroborando com o estudo bibliométrico de Zott, Amit e 
Massa (2011), que fizeram um amplo levantamento do uso do conceito de modelo de negócios em pesquisas divulgadas desde 1995, em 1.177 artigos, de periódicos de impacto mundial, como Academy of Management Journal (AMJ), Academy of Management Review (AMR), Administrative Science Quarterly (ASQ), Journal of Management (JOM), Journal of Management Studies (JMS), Management Science (MS), MIS Quarterly, Organization Science (OS) e Strategic Management Journal (SMJ).

Essa escala possibilitará o estudo dos modelos de negócios em pesquisas de natureza quantitativa, ainda raros, segundo os mesmos autores. Vários foram os estudos qualitativos sobre modelo de negócios nas empresas, mas os resultados desses estudos trazem um conhecimento ainda limitado e de difícil generalização, embora tenham permitido algumas ferramentas de análise, como o Canvas, desenvolvido por Osterwalder e Pigneur (2010).

Ressalta-se que a escala desenvolvida nesta pesquisa deve ser aplicada em outras amostras, visando à sua consolidação e possíveis ajustes futuros. Como sugestão, as próximas pesquisas podem utilizar análises fatoriais confirmatórias, como forma de revalidação da escala, e a utilização destas para a análise multivariada, por meio da ANOVA, MANOVA, regressões e modelagem em equações estruturais.

Quanto à operacionalização dos constructos, a Proposta de Valor (PS_PV), em consonância com as recomendações teóricas, deve ser testada como variável dependente do modelo de negócios. Os demais constructos - Segmentação de Mercado (IC_SM), Canais de Distribuição (IC_CD), Relacionamento com o Cliente (IC_RC), pertencentes à dimensão Interface com o Cliente, e os constructos Capacidades (GI CP) e Parceria (GI_PA), que pertencem à Gestão da Infraestrutura teriam os seus impactos na Proposta de Valor, mensurados e testados. Novas pesquisas desenvolvidas nessas linhas servirão para desenvolver e consolidar o conhecimento a respeito dos modelos de negócios e para a eliminação das deficiências relatadas por Teece (2010) no uso dos modelos de negócios. Segundo o autor, esses modelos são raramente 
analisados e frequentemente mal compreendidos, com pouca atenção dada ao seu design. Ele reconhece explicitamente que o aumento da compreensão da essência dos modelos de negócio deve ajudar a compreensão de uma variedade de assuntos, incluindo comportamento do mercado, concorrência, inovação, estratégia e vantagem competitiva.

\section{Considerações finais}

Este trabalho teve por objetivo construir e validar uma escala para mesurar as variáveis que definem o conceito de modelo de negócios, inédita, conforme levantamento bibliográfico e o estudo bibliométrico de Zott, Amit e Massa (2011).

A proposta inicial da pesquisa contemplava a utilização de 34 variáveis para a escala, porém, após os procedimentos de validação, cinco variáveis foram excluídas: PS_PV4; PS_PV5; IC_RC3; GI_CV3 e GF_MR1. As variáveis GI_CV1 e GI_CV2 agruparam-se ao constructo "Capacidades", em vez de "Configuração de Valor", o que provocou a dissolução deste.

Embora este estudo contribua para que se avance na compreensão dos modelos de negócios, inclusive pela possibilidade da realização de estudos de causa e efeito, estes estão submetidos a limitações que merecem registros. A primeira delas se refere à amostra que foi predominante da cidade de Fortaleza-CE. A utilização do questionário eletrônico teve como finalidade alcançar respostas de outros estados brasileiros, mas o retorno de outras unidades federativas foi de apenas $10 \%$ do total de observações coletadas. Outra limitação reside no fato de que cerca de um terço dos respondentes não ocupava cargos de alta direção, tendo, portanto, menos experiência profissional e/ou de gestão, gerando maior propensão a erros em decorrência da dificuldade de compreensão das assertivas do questionário.

Metodologicamente, sugere-se o uso de outras técnicas estatísticas, como MANOVA, análise discriminante e regressão logística, tendo a proposta de valor como variável dependente, e a aplicação da modelagem em equações estruturais. 


\section{Referências}

aMIT, R.; ZOTT, C. Value creation in e-business. Strategic Management Journal, v. 22, n.6/7, p. 493-520, June - July 2001.

ANDERSON, E.; DAY, G.; RANGAN, V. K. Strategic Channel Design. Sloan Management Review, v. 38, n. 4, Jul., 1997.

ANDREWS, P. P.; HAHN, J. Transforming supply chains into value webs. Strategy \& Leadership, v. 26, n. 3, p. 6-11, 1998.

ARAÚJO, J. B.; ZILBER, S. N. Adoção de E-Business e Mudanças no Modelo de Negócios: inovação organizacional em pequenas empresas dos setores de comércio e serviços. Gestão \& Produção, São Carlos/ SP, v. 20, n. 1, p.147-163, Jan./Mar. 2013.

BAGCHI, S.; B. TULSKIE. E-business Models: Integrating Learning from Strategy Development Experiences and Empirical Research. In: Annual International Conference of the Strategic Management Society, $20^{\text {th }}$, 2000, Vancouver/BC. Anais ..., Vancouver, 2000. p. 1-16.

BLATTBERG, R.; GETZ, G.; THOMAS, J. S. Customer Equity: building and managing relationships as valuable assets. Boston: Harvard Business School Press, 2001.

CHASTON, I; MANGLES, T. E-Commerce in Small US Manufacturing Firms: a pilot study on internal competencies. Journal of Marketing Management, v. 18, n. 3/4, p. 341-360, Fev. 2002.

CHESBROUGH, H. Business model innovation: Opportunities and barriers. Long Range Planning, v. 43, n. 3/4, p. 354-363, April-June 2010.

COSTA, F. J. Mensuração e Desenvolvimento de Escalas: aplicações em administração. Rio de Janeiro: Editora Ciência Moderna, 2011.

ETHIRAJ, S.; GULER, I.; SINGH, H. The impact of Internet and electronic technologies on firms and its implications for competitive advantage. The Wharton School. Research paper, 2000. Disponível 
em: <http://www.academia.edu/3764792/The_impact_of_Internet and_electronic_technologies_on_firms_and_its_implications_for_ competitive_advantagehttp://knowledge.emory.edu/papers/977.pdf>. Acesso em: 17 ago 2013.

GRANT, R. M. The Resource-Based Theory of Competitive Advantage: implications for strategy formulation. California Management Review, v. 33, n. 3, p. 114-135, April 1991.

HAIR, J. F.; BLACK, W. C.; BABIN; B. J.; TATHAM; R. L. Análise Multivariada de Dados. 6a ed. Porto Alegre: Bookman, 2009.

JOIA, L. A.; FERREIRA, S. Modelo de Negócios: constructo real ou metáfora de estratégia? Cadernos EBAPE, Rio de Janeiro, v. 3, n. 4, p. 1-18, Dez. 2005.

MAGRETTA, J. Why business models matter. Harvard Business Review, v. 80, n. 5, p. 86-92, May 2002.

MANSFIELD, G. M.; FOURIE, L. C. H. Strategy and business models strange bedfellows? Acase for convergence and its evolution into strategic architecture. South African Journal of Business Management, v.35, n.1, p.35-44, March 2004.

NALEBUFF, B.; BRANDENBURGER; A. Co-opetition: Competitive and cooperative business strategies for the digital economy. Strategy \& Leadership, v. 25, n. 6, p. 23-28, Nov./Dec. 1997.

OSTERWALDER, A. The Business Model Ontology: a proposition in a design science approach. 2004, 172 f. Tese (Doutorado em Gestão da Informática). Universidade de Lousanne, Suiça, 2004.

OSTERWALDER, A; PIGNEUR, Y. Business Model Generation. New Jersey: John Wiley \& Sons Inc., 2010.

- Designing business models and similar strategic objects: the contribution of IS. Journal of the Association for Information System, v. 14 , n. 5 , p. 237-244, May 2013. 
; PIGNEUR, Y.; TUCCI, C. L. Clarifying Business Models: Origins, Present, and Future of the Concept. Communications of the Association for Information System, v. 15, Maio 2005.

PITT, L.; BERTHON, P.; BERTHON, J. P. Changing Channels: The Impact of the Internet on Distribution Strategy. Business Horizons, v.42, n. 2, p. 19-28, March-April 1999.

PORTER, M. Strategy and the internet. Harvard Business Review, v. 79, n. 2, p. 63-78, March 2001.

RAPPA, M. A. The utility business model and the future of computing services. IBM Systems Journal, v.43, n.1, p. 32-42, January 2004.

SANTOS, R. S.; SELOTI JR; S. L.; SILVA, R. J. R. Changing BusinesS Model Traditional vs Gourmet Bakeries. In: Iberoamerican Academy Conference, $8^{\text {th }}$, 2013, São Paulo/SP. Anais... São Paulo: Escola de Administração de Empresas de São Paulo - EAESP - FGV, Dezembro, 2013. p. 1-16.

SEDDON, P. B.; LEWIS, G. P.; FREEMAN, P.; SHANKS, G. The case for viewing business models as abstractions of strategy. Communications of the Association for Information Systems, v. 13, Article 25, Abril, p. 2004.

SELZ, D. Value Webs: emerging forms of fluid and flexible organizations.1999. 229 f. Tese. (Doutorado em Ciência da Computação). University of St. Gallen, Suiça, 1999.

SERRANO, D. P. Geração X, Geração Y, Geração Z. Portal do Marketing. Disponível em: <http://www.portaldomarketing.com. br/Artigos/Geracao_X_Geracao_Y_Geracao_Z.htm> Acesso em: 20/02/2014.

STABELL, C. B.; FJELDSTAD, O. D. Configuring value for competitive advantage: on chains, shops, and networks. Strategic Management Journal, v. 19, n. 5, p. 413-437, May 1998. 
TAPSCOTT, D.; TICOLL, D.; LOWI, A. Digital Capital: harnessing the power of business webs. Boston: Harvard Business School Press, 2000.

TEECE, D. Business models, business strategy and innovation. Long Range Planning, v. 43, n.3/4, p. 172-194, April-June 2010.

WALLIN, J. Operationalizing Competences. In: Competence Perspective on Managing Internal Process. Advances in Applied Business Strategy, v. 7, Emerald Group, p. 151-179, August 2005312 p.

WERNEFELT, B. A resource-based view of the firm. Strategic Management Journal, v. 5, n. 2, p. 171-181, April/June 1984.

WU, F.; MAHAJAN, V.; BALASUBRAMANIAN, S. An analysis of e-business adoption and its impact on business performance. Journal of the Academy of Marketing Science, v. 31, n. 4, p. 425-447, October 2003.

ZHU, K.; KRAEMER, K. L.; XU, S. The process of innovation assimilation by firms in different countries: a technology diffusion perspective on e-business. Management Science, v. 52, n. 10, p. 1557-1576, October 2006.

ZOTT, C.; AMIT, R.; MASSA, L. The Business Model: Recent Developments and Future Research. Journal of Management, v. 37, n. 4, p. 1019-1042, July 2011.

Artigo recebido em: 27/10/2014

Aprovado em: 10/12/2014 